\title{
Search for dense molecular gas in two QSO host galaxies (Research Note)
}

\author{
J.-U. Pott ${ }^{1,2}$, A. Eckart ${ }^{2}$, M. Krips ${ }^{3}$, L. E. Tacconi-Garman ${ }^{1}$, and E. Lindt ${ }^{2}$ \\ 1 European Southern Observatory (ESO), Karl-Schwarzschildstr. 2, 85748 Garching bei München, Germany \\ e-mail: jpott@eso.org \\ 2 I. Physikalisches Institut, University of Cologne , Zülpicher Str. 77, 50937 Köln, Germany \\ ${ }^{3}$ Smithsonian Astrophysical Observatory, Submillimeter Array, HI 96720, USA
}

Received 21 February 2006 / Accepted 22 May 2006

ABSTRACT

\begin{abstract}
Context. The $\mathrm{HCN}(1-0)$ rotational line transition traces the dense $\left(n_{\mathrm{H}_{2}}>10^{4} \mathrm{~cm}^{-3}\right)$ fraction of the molecular gas typically located in starforming (SF) regions. In addition, an abnormally high $\mathrm{HCN} / \mathrm{CO}$ line ratio close to AGNs may indicate then conditions of an X-ray-dominated region. Observed correlations between the CO-, HCN-, and FIR luminosities in nearby non-active, starburst, and low-luminosity active galaxies represent the physical connection between star formation and molecular gas as its fuel. $\mathrm{HCN}(1-0)$ has hardly been investigated in nearby high-luminosity AGN within this context.

Aims. The aim of this study is to compare the HCN luminosity with published CO and IR luminosities to investigate the role of SF in the observed QSO host galaxies.

Methods. We used the IRAM $30 \mathrm{~m}$ for the first time to search for the $\mathrm{HCN}(1-0)$ transition in two standard QSO host galaxies at $z \sim 0.1$.

Results. Our upper limits on $L_{\mathrm{HCN}}$ agree with the known correlations and do not show strong excess abundance or excitation of the $\mathrm{HCN}$ due to the luminous active quasar nucleus. The starburst origin of the far-infrared luminosity in the observed QSO hosts cannot be proven unambiguously by the upper limits. We found that the IR/FIR ratio indicates independently of $L_{\mathrm{IR}}$ if a significant amount of AGN heated dust is present.
\end{abstract}

Key words. quasars: general - galaxies: ISM - ISM: molecules

\section{Introduction}

The evolutionary sequence from ultraluminous infrared galaxies (ULIRGs; $L_{\mathrm{IR}} \geq 10^{12} L_{\odot}$ ) to quasars has become a popular, though controversial, theory (e.g. Sanders et al. 1988; Sanders 2003). Common IR properties tracing active galactic nuclei (AGN) and star formation (SF) in the respective galaxies support such evolutionary schemes. (Sub-)mm photometry and spectroscopy have very recently revealed that optically luminous quasars already show in the early universe $(z>1)$ indications for vigorous star formation, ULIRG-like FIR/CO luminosity ratios, and HCN-emission (Cox et al. 2005; Solomon \& vanden Bout 2005, and references therein). The dust emission appears to trace the evolutionary history of quasars (Haas et al. 2003). Optically selected standard quasars (e.g. the PG quasars) have NIR-MIR luminosities dominated by the AGN with negligible stellar light contributions at wavelengths shorter than $5 \mu \mathrm{m}$. Nevertheless SF seems to be present in quasar hosts (Surace et al. 2001).

Haas et al. (2003) discuss the possibility of quantifying the star burst (SB) contribution to the infrared luminosities of PG quasars on the basis of sensitive ISO SEDs. The radio-FIR correlation known from SB galaxies (Condon 1992) appears to be fulfilled for the radio-quiet PG quasars, while the IR brightest $\left(L_{\text {FIR }} \geq 10^{12} L_{\odot}\right)$ radio-loud counterparts do not clearly indicate the importance of SB contribution to the FIR. In these IR-brightest quasars, the FIR component might simply constitute the Rayleigh-Jeans tail of the SED of the AGN-heated dust, normally dominating only the MIR emission of the quasar hosts.
Recent CO studies of QSOs (e.g. Evans et al. 2001) have revealed large amounts of molecular gas by applying the adequate conversion factors between $L_{\mathrm{CO}}$ and the total molecular gas mass. The QSOs show systematically higher infrared-to-CO luminosity ratios compared to the majority of galaxies with similarly high infrared luminosity, but no visible AGN. Evans et al. (2001) conclude that their selected sources show either a strong AGN-heated dust contribution to $L_{\mathrm{IR}}$ or an increase in star formation efficiency (SFE), i.e. star formation rate (SFR) with respect to the total amount of molecular gas available.

Solomon et al. (1992) and Gao \& Solomon (2004, hereafter G04) studied the HCN content in 65 nearby galaxies, covering three orders of magnitude of $L_{\mathrm{IR}}$ up to the ULIRG regime and established that the dense gas tracer $\mathrm{HCN}$ is tightly correlated to the SFR. Furthermore, the global HCN/CO line-intensity ratio appears to be a strong starburst indicator. By applying this putative SB indicator to their entire sample, including the most infrared luminous objects, the far-infrared luminosity could be mostly assigned to starburst activity, with only an insignificant contribution from AGN heated dust.

The question of the SB contribution to the total $L_{\mathrm{IR}}$ still remains for infrared-bright QSO+hostgalaxy systems, which harbor a strong AGN. Their FIR may comprise a major SB contribution and follow SB-induced FIR/CO and FIR/HCN correlations. To probe the latter correlation, we did a spatially unresolved search for $\operatorname{HCN}(J=1-0)$ emission in two PG quasars (Sect. 2), bearing in mind that the weak global HCN fluxes of QSOs might be significantly enhanced by direct AGN excitation. The results are presented in Sect. 3 In Sect. 4 the results are discussed and 
Table 1. Source list and parameters of the observing run in June 2005 with the IRAM 30 m-telescope. At $80 \mathrm{GHz}$, the telescope properties are: HPBW: 31"; Beam efficiency: 0.8; forward efficiency: 0.95; $\mathrm{S} / T_{\mathrm{mb}}$ : $4.95 \mathrm{Jy} / \mathrm{K}$.

\begin{tabular}{ccccccccccc}
\hline \hline Source & $\begin{array}{c}\text { RA } \\
(\mathrm{J} 2000)\end{array}$ & $\begin{array}{c}\text { Dec } \\
(\mathrm{J} 2000)\end{array}$ & $z_{\mathrm{CO}}$ & $\begin{array}{c}D_{L^{a}}{ }^{2} \\
{[\mathrm{Mpc}]}\end{array}$ & Transition & $\begin{array}{c}\text { Sky freq. } \\
{[\mathrm{GHz}]}\end{array}$ & $\begin{array}{c}\log L_{\mathrm{IR}}{ }^{b} \\
{\left[\log L_{\odot}\right]}\end{array}$ & $\begin{array}{c}\log L_{\mathrm{FIR}}{ }^{b} \\
{\left[\log L_{\odot}\right]}\end{array}$ & $\begin{array}{c}F_{\mathrm{CO}}^{\text {average }} \\
{\left[\mathrm{mJy}^{2}\right]}\end{array}$ & $\begin{array}{c}L_{\mathrm{CO}}^{\prime}{ }^{a} \\
{\left[\mathrm{~K} \mathrm{~km} \mathrm{~s} \mathrm{pc}^{-1}\right]}\end{array}$ \\
\hline PG0838+770 & 084445.36 & 765309 & 0.132 & 540 & $\mathrm{HCN}(J=1-0)$ & 78.296 & 11.48 & 11.15 & 37 & $2.1 \times 10^{9}$ \\
PG1415+451 & 141700.84 & 445606 & 0.114 & 470 & $\mathrm{HCN}(J=1-0)$ & 79.562 & 11.39 & 10.83 & 66 & $1.5 \times 10^{9}$ \\
\hline
\end{tabular}

${ }^{a}$ from Evans et al. (2001); ${ }^{b}$ calculated from the IRAS flux densities as published by Sanders et al. (1990) using the standard IRAS conversion formulae (e.g. presented in Table 1 in Sanders \& Mirabel 1996). $L_{\mathrm{IR}}$ covers 8-1000 $\mu \mathrm{m}$ and $L_{\mathrm{FIR}}$ covers $40-500 \mu \mathrm{m}$.

compared with published data. The conclusions are briefly summarized in Sect. 5.

\section{Observations and source selection}

The observational details are given in Table 1 . The weather conditions were good with system temperatures of 150-250 K and zenith opacities of $0.05 \leq \tau_{\text {zen }} \leq 0.1$ at the sky frequencies. Because the observing frequencies fall outside the standard tuning range of the receivers, we successfully conducted a frequency tuning test on the giant molecular cloud Sgr B2. Pronounced lines like ${ }^{13} \mathrm{CH}_{3} \mathrm{OH}, \mathrm{H}^{13} \mathrm{CCCN}$, and EtCN were found to coincide with the findings of the molecular line survey of Turner $(1989,1991)$ of similar spectral resolution.

Each line was resolved by $1 \mathrm{MHz}$-filter bank backend, while the data was smoothed to a velocity resolution of about $25 \mathrm{~km} \mathrm{~s}^{-1}$. Frequent observations of continuum sources provided a pointing accuracy of 3-5". We used single-sideband SIS receivers and the wobbler with a switch cycle of $2 \mathrm{~s}$ and a beam throw of $110^{\prime \prime}$. At least 4 times per hour, we performed a chopper wheel calibration. Baselines were flat, and only linear polynomials were used for correction.

We selected the two sources from a sample of 13 PG quasars with known CO fluxes (Evans et al. 2001; Scoville et al. 2003) that show the brightest average CO flux density (lineflux/linewidth; Table 1).

\section{Results - upper limits on the HCN luminosity}

The observational results are presented in Fig. 1 and Table 2. The spectra in Fig. 1 are smoothed to a resolution adapted to the CO data, which are presented by Evans et al. (2001) assuming a similar width of the HCN line. Under the same assumption, we calculated $3 \sigma$ upper limits to the luminosity of the $\operatorname{HCN}(J=$ $1-0)$ rotational line transition (Table 2). Accounting for the weak expected line fluxes, our $\sigma$ are lower by a factor of five than the average rms on the $30 \mathrm{~m}$ line fluxes achieved by G04.

\section{Discussion of the results}

In the following, the observational results are compared to published $\mathrm{CO}$ and FIR luminosities and the resulting ratios are discussed.

\section{1. $\mathrm{HCN} / \mathrm{CO}$ line ratio}

The upper limits on the HCN luminosity lead to $\mathrm{HCN} / \mathrm{CO}$ line ratios of less than $\sim 0.46$. These global ratios are around the highest ratios measured close to low-luminosity AGN (e.g. Helfer \& Blitz 1995, report $\mathrm{HCN} / \mathrm{CO} \approx 0.6$ in the nucleus of the Seyfert 2 prototype NGC 1068). Kohno (2005) reports that ratios above 0.2 can appear in the nuclei of some Seyferts most
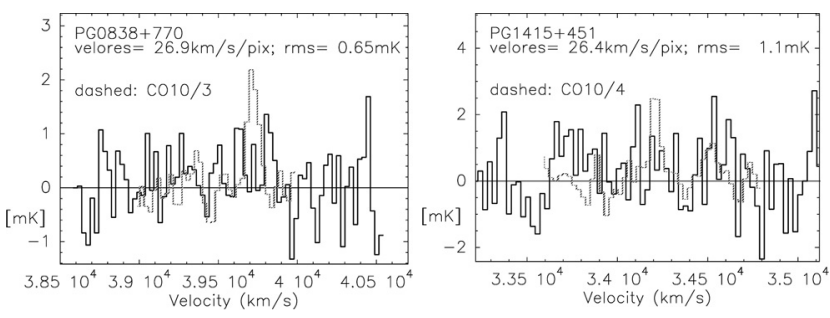

Fig. 1. The spectra of the two QSO hosts towards which we searched for the HCN $(1-0)$ emission. The velocity scale follows the optical convention $v=c z$. The flux density is given in the $T_{\mathrm{mb}}$-scale. The spectra have been smoothed to a resolution of $14 \mathrm{MHz}$ with a $7 \mathrm{MHz}$ sampling. The derived $3 \sigma$-upper limits are given in Table 2 . We overplotted the $\mathrm{CO}(J=1-0)$ spectra from Evans et al. (2001) converted to the $30 \mathrm{~m}-$ $T_{\mathrm{mb}}$-scale assuming that the QSOs had not been spatially resolved by their interferometric observations.

Table 2. The observational results.

\begin{tabular}{ccccc}
\hline \hline Source & $\begin{array}{c}\Delta v_{\mathrm{FWHM}}{ }^{a} \\
{\left[\mathrm{~km} \mathrm{~s}^{-1}\right]}\end{array}$ & $\begin{array}{c}S_{\mathrm{HCN}} \Delta v^{a} \\
{\left[\mathrm{Jy} \mathrm{km} \mathrm{s}^{-1}\right]}\end{array}$ & $\begin{array}{c}L_{\mathrm{HCN}}^{\prime}{ }^{b} \\
{\left[\mathrm{~K} \mathrm{~km} \mathrm{~s}^{-1} \mathrm{pc}^{2}\right]}\end{array}$ & $L_{\mathrm{HCN}}^{\prime} / L_{\mathrm{CO}}^{\prime}$ \\
\hline PG0838 & 90 & $<0.67$ & $<7.2 \times 10^{8}$ & $<0.34$ \\
PG1415 & 50 & $<0.84$ & $<6.9 \times 10^{8}$ & $<0.46$ \\
\hline
\end{tabular}

${ }^{a} 3 \sigma$ flux limits with $\sigma\left(S_{\mathrm{HCN}} \Delta v\right)=\sigma\left(S_{\text {chan }}\right) \cdot\left(2 \Delta v_{\mathrm{FWHM}} \Delta v_{\text {chan }}\right)^{1 / 2}$, assuming a triangular line shape; $\sigma\left(S_{\text {chan }}\right)$ and velocity resolution $\Delta v_{\text {chan }}$ are given in Fig. 1.

probably due to intense X-ray irradiation by the AGN. Such Xray dominated regions (XDRs) appear to be confined to the innermost regions $(\$ 100 \mathrm{pc})$, in general without significant contributions to the global HCN luminosity in gas-rich host galaxies (Kohno et al. 2003; Usero et al. 2004).

Although the spatially unresolved IRAM 30 m observations can only probe the global $L_{\mathrm{HCN}}$, the outstanding nuclear activity in quasars might contribute significantly to the global HCN excitation, too. Thus our upper limits on the global line ratios can exclude a strong global $\mathrm{HCN} / \mathrm{CO}$ excess in the QSO hosts beyond the values found in low-luminosity active nuclei. But since our upper limits are above 0.3 , it cannot be ruled out that a significant part of the total HCN is XDR-like excited by the AGN itself.

A comparison of the reached sensitivities (Fig. 1) with the respective $\mathrm{HCN} / \mathrm{CO}$ line ratios in both observed QSOs might suggest, however, that the higher $\mathrm{HCN} / \mathrm{CO}$ upperlimit of PG 1415+451 derives from the lower sensitivity reached only in this observation.

\subsection{Probing the FIR origin}

In infrared luminous QSO host galaxies, the IR is reprocessed UV-optical irradiation that is emitted by the AGN itself (AGN-scenario) and by massive stars (SF-scenario). 


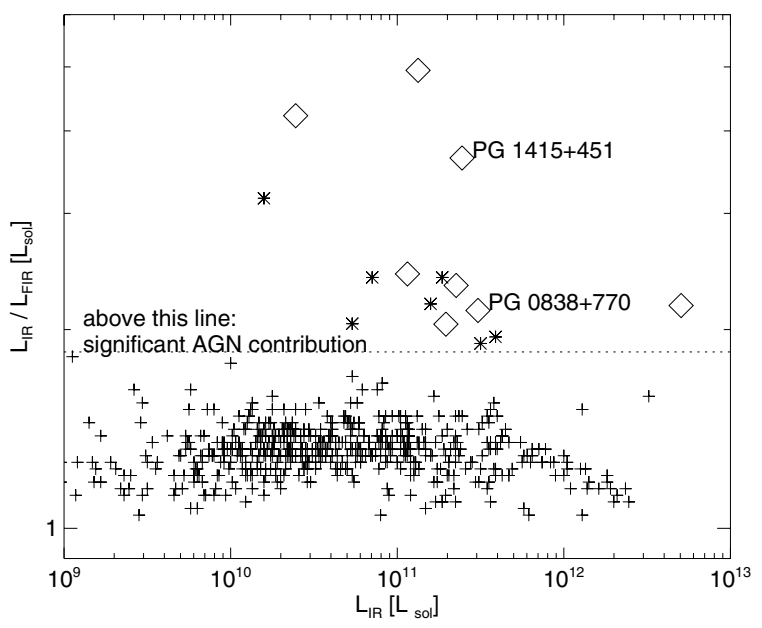

Fig. 2. Here we introduce the IR/FIR ratio as a diagnostic to reveal significant AGN-contribution to the total infrared luminosity of a galaxy. The IRAS Revised Bright Galaxy Sample (Sanders et al. 2003) is plotted, including virtually all galaxies from G04 (crosses). The dotted line represents IR/FIR $=1.85$ and is arbitrarily chosen to guide the reader's eye. All radio-quiet PG quasars (including those observed here; the IRAS flux densities taken from Sanders et al. 1990) are above the line and indicated by diamonds. The stars above the line are active galaxies of Seyfert type. There are no quiescent/SB galaxies above the line. We suggest using such a plot as a diagnostic to find significant AGN-contributions to the IR luminosity of a galaxy (see Sect. 4.2). The size of the symbols reflect the order of magnitude of the luminosity uncertainties.

Multiwavelength information is required to investigate which scenario dominates the global properties of a specific galaxy, or whether neither dominates.

We plotted in Fig. 2 the IR/FIR luminosity ratio of a large flux-limited sample of galaxies - the IRAS bright galaxy sample (IBGS) includes normal, starburst, active, and ultraluminous IR galaxies (Sanders et al. 2003) - together with the radio-quiet subset of PG quasars (published in Sanders et al. 1990). Nearly all galaxies of the G04-sample are included in the IBGS, which comprises all levels of starburst activity. The vast majority of the galaxies populates a continuous band around IR/FIR $\sim 1.3$ over several orders of magnitude of total IR luminosity, whereas all quasar hosts, including the observed ones and a few Seyferts, are located well above this value.

This demonstrates that the AGN dominates the global $L_{\mathrm{IR}}$ in our quasars. Thus we concentrate on the FIR wavelength regime in the following. How strong is the FIR luminosity (Table 1) affected by the AGN? We can investigate the AGN-powered fraction of the FIR ( FIR $\left._{\mathrm{AGN}}\right)$ by a comparison with the molecular gas luminosities.

We plotted the FIR/CO- and the FIR/HCN-luminosities of the G04-sample in Fig. 3 (upper and lower panel, resp.). We probed for linear correlations by $\chi^{2}$-fits to slopes in log-space, the estimated slopes are given in Fig. 3. We indeed found slopes of one for the quasars and the G04-subsample excluding ULIRGs for the FIR/CO correlation. The IR/gas correlations, published by G04, translate into similar FIR/gas correlations, reflecting the luminosity-independent IR/FIR ratios of the sample (Fig. 2). With respect to the linear correlation given in Fig. $3\left(L_{\mathrm{FIR}}=26 L_{\mathrm{CO}}^{\prime}\right)$, the quasars show an FIR excess by a factor of 2.6 (PG 0838) and 1.7 (PG 1415). These excesses can limit FIR AGN $_{\text {, if we assume (i) normal SFE (i.e. FIR }} \geq 26 L_{\mathrm{CO}}^{\prime}$ ) and (ii) that the detected $\mathrm{CO}$ is predominantly fueling SF.
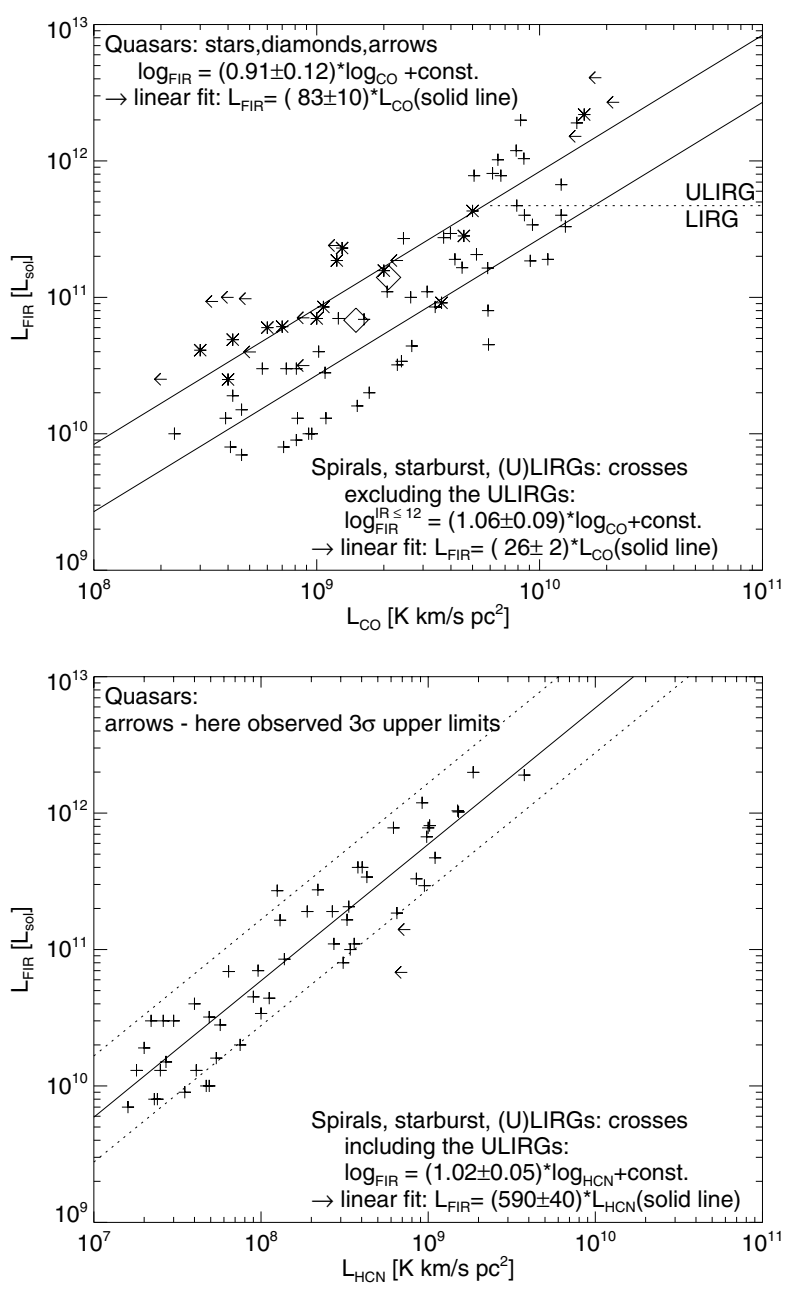

Fig. 3. The correlation plots of G04 extended by quasar hosts with known CO luminosities (Alloin et al. 1992; Evans et al. 2001; Scoville et al. 2003, the two investigated quasars are highlighted as diamonds) (upper panel) and the estimated upper limits (arrows) on $L_{\mathrm{HCN}}$ (lower panel). Linear correlations are given (solid lines), resulting from a fit in log-space, where the slope was fixed to one. The dotted lines in the lower panel are showing the expected FIR/HCN correlation for quasars in case of the AGN-scenario (Sect. 4.2), for global $\mathrm{HCN} / \mathrm{CO}$ ratios of 0.05 (upper line) and 0.3 (lower line). In contrast, the SF-scenario implies that the quasars are predicted to follow the given FIR/HCN correlation. Note that the less sensitive upper HCN-limit (PG1415) is further away of the standard FIR/HCN correlation. Individual uncertainties are $30-50 \%$.

The FIR/CO ratio of our quasars is similar to or below the ratio of most ULIRGs. Since G04 argued that the increased FIR/CO ratio in ULIRGs could be evoked entirely by an increased SFE without significant FIR $_{\mathrm{AGN}}$, the lower limit

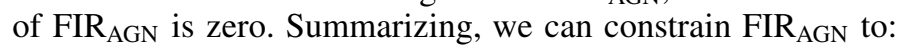
$0 \leq \mathrm{FIR}_{\mathrm{AGN}}(\mathrm{PG} 0838) \leq 40 L_{\mathrm{CO}}^{\prime}$ and $0 \leq \operatorname{FIR}_{\mathrm{AGN}}(P G 1415) \leq$ $20 L_{\mathrm{CO}}^{\prime}$.

Furthermore, the quasars with published $L_{\mathrm{CO}}$ show a linear FIR/CO correlation themselves (upper solid line in Fig. 3, upper panel). If we include the ULIRGs in the quasar sample assuming that they are dust-enshrouded quasars, the logarithmic slope increases to $(1.07 \pm 0.09)$; i.e. a linear correlation is still within the uncertainties. We also did not include the quasars with upper limits on their CO luminosities in the fits. Since they appear to be equally distributed above the considered quasars in Fig. 3, a significant change of the logarithmic slope is not to be expected 
from incorporating these objects. On the other hand, most of the ULIRGs are located above the linear correlation plotted in Fig. 3. This could argue for a logarithmic slope steeper than one if more ULIRGs with similar properties as the measured ones were included. But together with the CO-upperlimit quasars, a linear correlation shifted towards higher $L_{\mathrm{FIR}}$ is possible, too.

On the basis of this linear correlation, we can assume FIR $_{\mathrm{AGN}}$ of an average QSO to fulfill: $0 \leq$ FIR $_{\mathrm{AGN}} \leq 57 L_{\mathrm{CO}}^{\prime}$ following the above argumentation. Thus a global AGN dominance of the entire IR of the quasar host is possible. This AGN-scenario can lead to two different expectations for the global FIR/HCN ratio of quasars, depending on the global $\mathrm{HCN} / \mathrm{CO}$ ratio. Using the linear correlations given in Fig. 3, an $\mathrm{HCN} / \mathrm{CO}$ ratio of 0.05 (G04) would shift the FIR/HCN relation upwards to FIR $=1660 \mathrm{HCN}$ (upper dotted line). On the other hand, global AGN-dominance could imply a significant increase in the global $\mathrm{HCN} / \mathrm{CO}$ ratio (cf. Sect. 4.1). Assuming an increased $\mathrm{HCN} / \mathrm{CO}$ ratio of 0.3 would lead to FIR $=280 \mathrm{HCN}$ (lower dotted line).

If the SF-scenarios holds $\left(\mathrm{FIR}_{\mathrm{AGN}} \sim 0\right)$ instead, the quasars are expected to follow the standard correlation (solid line). Since even our more sensitive upper limit (PG0838) on FIR/HCN does not exclude any range of the expected FIR/HCN, more sensitive $\mathrm{HCN}$ data are needed to better constrain the FIR/HCN ratio in quasars.

Furthermore, the dotted lines in Fig. 3 are at the borders of the G04-sample. Since a similar scatter is to be expected for QSOs, only a statistically significant sample of $\mathrm{HCN}$-detected quasars could establish the existence of a nonstandard FIR/HCN correlation for quasars.

\section{Conclusions}

For the first time we can publish firm upper limits on the HCN $(J=1-0)$ rotational line emission of the host galaxies of two optically selected nearby standard QSO hosts from the PG sample. The derived upper limits on the global HCN-CO ratios of $\lesssim 0.4$ cannot rule out significant direct $\mathrm{HCN}$ excitation by the AGN in XDRs in the observed hosts.

Furthermore, we put the HCN upper limits in the context of previous statistical studies, investigating the molecular gas and IR properties of nearby spiral, SB and low-luminosity active galaxies. We propose to derive a significant AGN-contribution to the total infrared luminosity of a galaxy from an IR/FIR ratio significantly larger than 1.3 (as found in the observed quasars).

Our limits on FIR/HCN are consistent with the standard $L_{\mathrm{FIR}}-L_{\mathrm{HCN}}$ correlation of quiescent and SB galaxies, as well as with hypothetic correlations for AGN-dominated FIR to $\mathrm{HCN}$ in QSO hosts. We have shown that proving the existence of a HCN-FIR correlation in evolved quasar hosts is within observational reach.

Acknowledgements. We would like to acknowledge C. Kramer for his support. Further we are grateful to the referee for helping to improve the article significantly. The presented work is based on observations carried out with the IRAM $30 \mathrm{~m}$ telescope. IRAM is supported by the INSU/CNRS (France), MPG (Germany), and IGN (Spain). This research made use of the NASA/IPAC Extragalactic Database (NED), which is operated by the Jet Propulsion Laboratory, California Institute of Technology, under contract with the National Aeronautics and Space Administration. J.U.P. was funded by an ESO studentship.

\section{References}

Alloin, D., Barvainis, R., Gordon, M. A., \& Antonucci, R. R. J. 1992, A\&A, 265, 429

Condon, J. J. 1992, ARA\&A, 30, 575

Cox, P., Beelen, A., Bertoldi, F., et al. 2005, in The Dusty and Molecular Universe: A Prelude to Herschel and ALMA, 115

Evans, A. S., Frayer, D. T., Surace, J. A., \& Sanders, D. B. 2001, AJ, 121, 3285

Gao, Y., \& Solomon, P. M. 2004, ApJ, 606, 271

Haas, M., Klaas, U., Müller, S. A. H., et al. 2003, A\&A, 402, 87

Helfer, T. T., \& Blitz, L. 1995, ApJ, 450, 90

Kohno, K. 2005, in The Evolution of Starbursts, AIP Conf. Proc., 783, 203

Kohno, K., Ishizuki, S., Matsushita, S., Vila-Vilaró, B., \& Kawabe, R. 2003, PASJ, 55, L1

Sanders, D. B. 2003, J. Kor. Astron. Soc., 36, 149

Sanders, D. B., \& Mirabel, I. F. 1996, ARA\&A, 34, 749

Sanders, D. B., Soifer, B. T., Elias, J. H., et al. 1988, ApJ, 325, 74

Sanders, D. B., Phinney, E. S., Neugebauer, G., Soifer, B. T., \& Matthews, K. 1990, ApJ, 357, 291

Sanders, D. B., Mazzarella, J. M., Kim, D.-C., Surace, J. A., \& Soifer, B. T. 2003, AJ, 126, 1607

Scoville, N. Z., Frayer, D. T., Schinnerer, E., \& Christopher, M. 2003, ApJ, 585, L105

Solomon, P. M., Downes, D., \& Radford, S. J. E. 1992, ApJ, 387, L55

Solomon, P. M., \& vanden Bout, P. A. 2005, ARA\&A, 43, 677

Surace, J. A., Sanders, D. B., \& Evans, A. S. 2001, AJ, 122, 2791

Usero, A., García-Burillo, S., Fuente, A., Martín-Pintado, J., \& Rodríguez-Fernández, N. J. 2004, A\&A, 419, 897 